\title{
Methionine Sulfoxide Reductase A Mediates Dietary Restriction-Induced Lifespan Extension in Caenorhabditis elegans
}

\section{Justin Minnerly ${ }^{1 \#}$, Jiuli Zhang ${ }^{1 \#, ~ R e b e c a ~ A l d u n a t e ~}{ }^{2}$, Herbert Weissbach ${ }^{1}$ and Kailiang Jia ${ }^{1 *}$}

${ }^{1}$ Department of Biological Sciences and Center for Molecular Biology and Biotechnology, Florida Atlantic University, Jupiter, Florida, USA

${ }^{2}$ Escuela de Biotecnología, Universidad Santo Tomás, Santiago, Chile

"These two authors contributed equally to this work.

\begin{abstract}
Background: Methionine sulfoxide reductase $\mathrm{A}(\mathrm{Msr})$ is a well-studied antioxidant enzyme that has been found to be important for protecting cells against oxidative damage and regulating lifespan in several species. However, the role of MsrA in dietary restriction has not been examined. The authors evaluated the function of MsrA in dietary restriction-induced lifespan extension in Caenorhabditis elegans.

Methods: $C$. elegans loss-of-function msra mutant animals and wild type control animals were subjected to two widely used dietary restriction treatments, solid dietary restriction (sDR) and dietary restriction by liquid bacteria (BDR). The survival of the animals was evaluated and the data was statistically analyzed.

Results: The loss-of-function mutation of msra significantly suppressed the lifespan extension conferred by solid dietary restriction. By contrast, $m s r a$ was dispensable for lifespan extension resulting from dietary restriction by diluted bacteria in liquid.

Conclusion: msra-1 is a major factor in the sDR-induced lifespan extension. This result, coupled with the previous finding that MsrA mediates the effect of insulin-like signaling on lifespan extension, indicates an essential role of MsrA in the aging process in C. elegans.
\end{abstract}

Keywords: MsrA; Dietary restriction; Aging; Lifespan; C. elegans

\section{Introduction}

Dietary restriction (DR) is defined as a decrease in the nutrient uptake of an organism without causing malnutrition. DR is the most consistent and reproducible method used to increase lifespan and has been shown to do so in over twenty different species [1-3]. Although the effect of DR on life span has been extensively studied the exact mechanism by which DR acts is still not fully understood. However, there is considerable evidence that the lifespan extension seen in DR is due to a reduction in oxidative damage [4]. The modified free radical theory of aging proposed that reactive oxygen species (ROS) derived from oxygen are responsible for cellular damage associated with ageing. This could be due to a decrease in the generation of reactive oxygen species (ROS) and/or an increase in the cellular protective mechanisms against oxidative damage under conditions of DR. As example, rodents subjected to DR showed a decrease in the age-associated production of mitochondrial ROS and slower accumulation of oxidative damage [4]. In addition, there are several reports of life span extension, independent of $\mathrm{DR}$, that have resulted from over-expression of enzymes such as superoxide dismutase (SOD) [5], catalase [6] and MsrA [7], which are known to protect cells against oxidative damage either by destroying ROS or, as in the case of MsrA, repairing damage to proteins due to methionine oxidation [8]. The MSR system is unique in that it can also function as part of an ROS scavenger system in which methionine residues in proteins can function as catalytic antioxidants [9].

The methionine sulfoxide reductase system is a highly conserved system [10]. Methionine residues are very susceptible to oxidation by ROS and are converted to either the $\mathrm{S}$ epimer of methionine sulfoxide (Met-S(o)) or the R-epimer (Met-R(o)) when chemically oxidized by ROS. The methionine oxidation can be reversed by the methionine sulfoxide reductase system, MsrA and MsrB $[8,10]$. MsrA reduces the $\mathrm{S}$ epimer of $\operatorname{Met}(\mathrm{o})$ in proteins and $\mathrm{MsrB}$ is specific for the $\mathrm{R}$ epimer of $\operatorname{Met}(0)$ [11-15].
Several studies have provided evidence that MsrA is important in protecting cells against oxidative damage and in the aging process. Eschericia coli and yeast mutants lacking MsrA have an increased sensitivity to oxidative stress [16-18]. Compared to wild type mice, MsrA-/- mice also show decreased resistance to oxidative stress $[19,20]$. Moreover, expression of both MsrA and MsrB declines in senescent human fibroblasts cells compared to young cells, and this decline is associated with accumulation of oxidized proteins [21]. Interestingly, over-expression of MsrA lowers the levels of ROS in cells [22] and increases lifespan in fruit flies and yeast $[7,23]$.

In C. elegans, axenic medium imposes dietary restriction (ADR) and extends C. elegans lifespan [24]. ADR was found to cause higher activities of the antioxidant enzymes SOD and catalase [24]. C. elegans fed with a dilution of bacteria in liquid medium (bacterial dietary restriction: BDR) showed an increased lifespan, which was dependent on PHA-4, a FOXA transcription factor [25]. Interestingly, PHA-4 was found to mediate BDR-induced longevity by upregulating SOD [25]. Recently, a C. elegans homolog of MsrA has been identified [26] and an $m s r a$ deletion mutant was reported to be sensitive to oxidative stress [27]. Moreover, this deletion mutation of msra decreases the lifespan of the long-lived daf-2 mutant worms [27]. Interestingly, the DAF$16 /$ FOXO3 transcription factor, which is a major target of the daf-2

*Corresponding author: Kailiang Jia, Ph.D., Department of Biological Sciences Center for Molecular Biology and Biotechnology, Florida Atlantic University, 5353 Parkside Dr. Jupiter, FL 33458, USA, Tel: 1-561-799-8054; Fax: 1-561-297-2749; E-mail: kjia@fau.edu

Received September 04, 2013; Accepted September 24, 2013; Published September 26, 2013

Citation: Minnerly J, Zhang J, Aldunate R, Weissbach H, Jia K (2013) Methionine Sulfoxide Reductase A Mediates Dietary Restriction-Induced Lifespan Extension in Caenorhabditis elegans. Aging Sci 1: 110. doi: 10.4172/2329-8847.1000110

Copyright: (c) 2013 Minnerly J, et al. This is an open-access article distributed under the terms of the Creative Commons Attribution License, which permits unrestricted use, distribution, and reproduction in any medium, provided the original author and source are credited. 
insulin-like signaling pathway, positively regulates msra expression [27]. However, the role of MsrA in DR has not been examined. Here we show that MsrA mediates the effect of solid dietary restriction on lifespan extension in C. elegans.

\section{Materials and Methods}

\section{C. elegans strains}

The Bristol N2 strain is the wild type $C$. elegans strain used in all experiments. Strains were maintained at $20^{\circ} \mathrm{C}$ as described by Brenner [28]. To obtain the homozygous msra-1(tm1421) mutant, N2 males were crossed to the msra-1(tm1421) II; lin-15B(n765ts) mutants. The outcross progeny were selected and self-propagated to remove the lin-15B(n765ts) mutation. Fifty msra- 1 mutant worms were examined for the homozygous deletion mutation by following a standard singleworm PCR [29] and all tested worms showed the homozygous deletion. The sequences of the primers used were:

\section{msra-1 F: 5' - CACATTTTGATTCCGCCCCGATT - 3';}

\section{msra-1 R: 5' - GACAACAACTCTTCAAATCCATT - 3'.}

\section{Solid dietary restriction (sDR) lifespan analysis}

The sDR method was modified from that previously described [30]. Briefly, the OP50 overnight culture was washed and re-suspended in $S$ Basal media without cholesterol. Serial dilutions were performed to achieve bacterial concentrations of $5.0 \times 10^{11}, 5.0 \times 10^{10}, 5.0 \times 10^{9}$, and $5.0 \times 10^{8}$ bacteria/ml. $250 \mu \mathrm{l}$ of these diluted bacterial cultures were spotted on each $60 \mathrm{~mm}$ plate on the day of transfer. 30 adult worms were placed on each plate containing various concentrations of bacteria starting at day 1 of adulthood. Three plates for each strain at each bacterial concentration were set up. In the first week of the lifespan experiments, DR plates that contain FUdR $(20 \mu \mathrm{g} / \mathrm{ml})$ were used to prevent progeny from hatching. Worms were transferred to fresh plates every other day. Dietary restriction (sDR) was considered $5 \times 10^{8}$ bacteria/ml and ad libitum (AL) was $5 \times 10^{11}$ bacteria $/ \mathrm{ml}[30]$. The nematode growth media (NGM) plates were modified by excluding peptone and increasing agar from $1.7 \%$ to $2.0 \%$. Carbenicillin $(50 \mu \mathrm{g} /$ $\mathrm{ml}$ ) was added to the plates to further prevent bacteria growth. The experiment was performed at $20^{\circ} \mathrm{C}$.

\section{Bacterial dietary restriction (BDR) lifespan analysis}

BDR was performed as previously reported [25,31]. To prepare liquid cultures for dietary restriction, an OP50 overnight culture grown at $37^{\circ} \mathrm{C}$ was washed and resuspended to adjust the bacterial concentration to $1.5 \times 10^{9}$ cells $/ \mathrm{ml}$ in $\mathrm{S}$ Basal media containing cholesterol $(5 \mathrm{mg} / \mathrm{ml})$, carbenicillin $(50 \mathrm{mg} / \mathrm{ml})$, tetracycline $(1 \mathrm{mg} /$ $\mathrm{ml})$ and kanamycin $(10 \mathrm{mg} / \mathrm{ml})$. Serial dilutions were performed to achieve bacterial concentrations of $7.5 \times 10^{8}, 1.5 \times 10^{8}, 7.5 \times 10^{7}, 2.5 \times 10^{7}$ and $5.0 \times 10^{6}$ bacteria/ml. Dietary restriction $(\mathrm{BDR})$ was considered $7.5 \times 10^{7}$ bacteria $/ \mathrm{ml}$ and ad libitum (AL) was $7.5 \times 10^{8}$ bacteria/ml [25]. To prevent progeny reproduction, FUdR was added at a concentration of $20 \mu \mathrm{g} / \mathrm{ml}$ in cultures during the first twelve days of lifespan analysis.

To subject animals to dietary restriction, well-fed 1-day old adult worms were transferred into liquid cultures at different bacterial concentrations. Liquid cultures were done in 12-well cell culture plates containing $1 \mathrm{ml}$ of culture per well. Each lifespan experiment consisted of 4 wells with 15 worms per well. The 12-well plates were placed on a gentle rocker at $20^{\circ} \mathrm{C}$ during the lifespan analysis. Survival of the animals was scored daily and worms were transferred to new cultures every 3-4 days. Animals were considered dead when they failed to respond to touch.

\section{Statistical Analysis}

GraphPad Prism 5 was used to generate survival curves and determine medians, means and percentiles. In all cases, P-values were calculated using the log-rank (Mantel- Cox) method.

\section{Results and Discussion}

Previous studies have shown that ADR and BDR extend C. elegans lifespan and up-regulate antioxidant enzymes [24]. PHA-4 (FOXA) was reported to mediate BDR-induced longevity by controlling SOD transcription [25]. Our goal was to determine whether MsrA may also be required for DR-mediated lifespan extension. A C. elegans msra1(tm1421) deletion mutant has been isolated [27], and we initially confirmed the homozygous deletion in the $m s r a-1$ mutant. Fifty $m s r a-1$ mutant worms were analyzed by using single worm PCR with primers flanking the deletion site. As expected, a $171 \mathrm{bp}$ deletion band was detected in all msra-1(tm1421) mutant worms, which confirmed the 908 bp deletion in the msra-1(tm1421) mutant (Figure S1).

Next, the msra-1(tm1421) mutant worms were subjected to dietary restriction to examine the possible role of $m s r a-1$ in DR-mediated lifespan extension. Various dietary restriction protocols have been developed in C. elegans such as decreasing food availability by bacteria dilution in liquid culture (BDR) [31] and on solid medium (sDR) [30]. Since DAF-16 is required for sDR-mediated lifespan extension [30] and msra- 1 is a target gene of DAF-16 [27], we initially examined the role of msra-1 in plate-based dietary restriction (sDR). Consistent with a previous report [30], the lifespan of N2 worms fed with $5 \times 10^{8}$ bacteria/ $\mathrm{ml}(\mathrm{DR})$ is significantly increased compared to that of animals fed with $5 \times 10^{11}$ bacteria/ml (AL: ad libitum). The mean lifespan is increased by $38 \%$ (from 21.25 days to 28.88 days; $\mathrm{P}<0.0001$, log-rank test) and the maximum lifespan is extended by 20 days (from 31 to 51 days) (Figure 1 and Table 1 ). The sDR- treated msra- 1 mutants have a slightly shorter (but significant) median life span than sDR-treated N2 animals (26.13 days v.s. 28.88 days, $\mathrm{P}=0.0002$ ). However, the most striking effect seen with the msra- 1 mutants was on the maximum lifespan. The maximum lifespan of sDR-treated msra-1 mutants is 15 days shorter than that of N2 worms (36 vs. 51 days) (Figure 1 and Table 1). It should be noted that the mean lifespan of sDR-treated msra-1 mutants is still increased

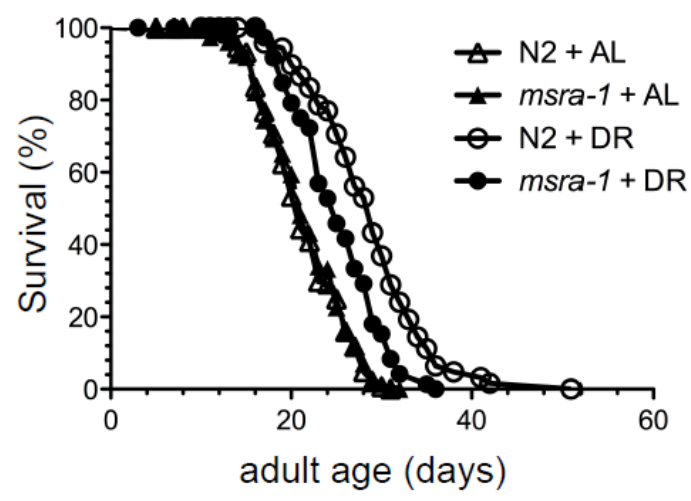

Figure 1: MSRA-1 mediates sDR-induced lifespan extension. Lifespan curves of wild- type $\mathrm{N} 2$ animals and msra1 mutant worms treated by SDR $\left(5 \times 10^{8}\right.$ bacteria/ml) and fed ad libtum $\left(5 \times 10^{11}\right.$ bacteria $\left./ \mathrm{ml}\right)$. N2 $+\mathrm{sDR}$, mean lifespan 28.88 days and maximum 51 days; $\mathrm{N} 2+\mathrm{AL}$, mean 21.25 days and maximum 31 days; msra-1 + sDR, mean 26.13 days and maximum 36 days; $m s r a-1+A L$, mean 21.38 days and maximum 32 days. This entire experiment was done twice and similar results were obtained. The pooled data from these two experiments are shown in this figure. 
Citation: Minnerly J, Zhang J, Aldunate R, Weissbach H, Jia K (2013) Methionine Sulfoxide Reductase A Mediates Dietary Restriction-Induced Lifespan Extension in Caenorhabditis elegans. Aging Sci 1: 110. doi: 10.4172/2329-8847.1000110

Page 3 of 5

Table 1: Statistical analysis of lifespan data.

\begin{tabular}{|c|c|c|c|c|}
\hline Strain and culture conditions & Mean lifespan (days) & Max.lifespan (days) & $\mathrm{N}^{1}$ & P Value ${ }^{2}$ \\
\hline \multicolumn{5}{|l|}{ sDR } \\
\hline $\mathrm{N} 2+5 \times 10^{11}$ cells $/ \mathrm{ml}(\mathrm{AL})$ & $21.25 \pm 0.6291$ & 31 & $145(31)$ & \\
\hline $\mathrm{N} 2+5 \times 10^{8}$ cells $/ \mathrm{ml}(\mathrm{DR})$ & $28.88 \pm 1.0078$ & 51 & $63(44)$ & $<0.0001$ (vs. N2 + AL) \\
\hline$m s r a-1+5 \times 10^{11}$ cells $/ \mathrm{ml}(\mathrm{AL})$ & $21.38 \pm 0.8985$ & 32 & $142(29)$ & 0.2083 (vs. N2 + AL) \\
\hline msra-1 + $5 \times 10^{8}$ cells $/ m l(D R)$ & $26.13 \pm 1.3901$ & 36 & $72(24)$ & 0.0002 (vs. N2 + DR) \\
\hline \multicolumn{5}{|l|}{ BDR } \\
\hline $\mathrm{N} 2+7.5 \times 10^{8}$ cells $/ \mathrm{ml}(\mathrm{AL})$ & $34.25 \pm 3.7666$ & 50 & $55(5)$ & \\
\hline $\mathrm{N} 2+7.5 \times 10^{7}$ cells $/ \mathrm{ml}(\mathrm{DR})$ & $42.5 \pm 1.8027$ & 55 & $49(11)$ & 0.0005 (vs. N2 + AL) \\
\hline msra-1 $+7.5 \times 10^{8}$ cells $/ \mathrm{ml}(\mathrm{AL})$ & $38.5 \pm 1.6583$ & 48 & $54(6)$ & \\
\hline$m s r a-1+1.5 \times 10^{8}$ cells $/ m l(D R)$ & $44.45 \pm 1.5562$ & 60 & $49(11)$ & $<0.0001$ (vs. $m s r a-1+\mathrm{AL}$ ) \\
\hline
\end{tabular}

${ }^{1} \mathrm{~N}=$ population size, numbers of censored animals are indicated in parenthesis;

${ }^{2} \mathrm{P}$ values (log- rank test) for mean life span of each group compared to group indicated in parentheses.

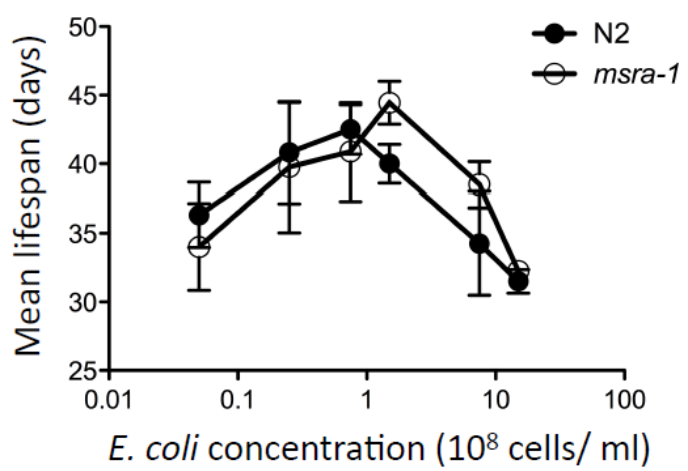

Figure 2: Dietary restriction using bacterial dilution (BDR) extends the lifespan of $m s r a-1(t m 1421)$ deletion mutants. BDR results in a parabolic curve for both wild-type worms (solid cycle) and msra-1(tm1421) mutant animals (open cycle). Error bars: s.e.m. The mean lifespan (44.45 days) of msra-1 mutant at optimal DR (1.5×10 bacteria/ml) is significantly longer than the mean lifespan (38.8 days) of worms fed ad libitum $\left(7.5 \times 10^{8}\right.$ bacteria/ml $)(p<0.0001$, log-rank test).

by 5 days compared to that of msra- 1 mutant worms fed ad libitum (from 21.38 days to 26.13 days, $\mathrm{P}<0.0001$ ), suggesting that the $m s r a-1$ mutation did not completely suppress the effect of sDR on the mean lifespan. As shown in Table 1 and Figure 1 the N2 and msra-1 mutants have a similar lifespan when fed ad libitum (mean life spans are 21.25 and 21.38 days, respectively, and the maximum lifespans are 31 and 32 days, respectively). This is in contrast to a previous study showing that msra-1 mutants lived 30\% shorter than N2 [27] when fed ad libitum. We were concerned about the difference in the results so the lifespan of msra-1 mutants fed ad libitum was examined in four independent trials and there was no obvious difference between $\mathrm{N} 2$ and msra- 1 mutant lifespans (Table S1). At present we cannot explain the different results obtained in the two studies. Regardless, the experiments demonstrate that the sDR-treated msra-1 mutants have both a shorter median and maximum lifespan.

In contrast to sDR, BDR extends C. elegans lifespan independently of DAF-16. If msra-1 mediates sDR-induced lifespan extension because it is a target gene of DAF-16, msra-1 might not be required for BDR to extend $C$. elegans lifespan. To test this, we subjected the msra-1 mutant and control wild type N2 worms to BDR. In agreement with previous reports [25,32], wild-type N2 animals are short lived at both high and very low bacterial concentrations. However, N2 mean lifespan is significantly increased at the optimal bacterial concentration (Figure 2 and Table 1). Similar to N2 worms, the msra-1 mutant worms also showed a parabolic curve with increased lifespan at the optimal bacterial concentration and short lifespan at both high and low bacterial concentrations (Figure 2 and Table 1 ). The results indicate that $m s r a-1$ mutants respond to BDR similar to wild-type N2 worms, suggesting msra-1 is dispensable for lifespan extension induced by BDR. It should be noted that the optimal bacterial concentration to maximize lifespan extension for msra- 1 mutants is $1.5 \times 10^{8}$ cells $/ \mathrm{ml}$, but it is $7.5 \times 10^{7}$ for N2 animals (Figure 2 and Table 1). Previous published studies have indicated that C. elegans with different genotypes can respond to DR differentially. Therefore, the concentration of bacteria in the food that maximizes lifespan for one genotype may be different from the one works for another genotype [32].

In C. elegans, dietary restriction has been mainly applied in four ways: dilution of bacteria in liquid medium (BDR) [31], eat-2 mutants with pharyngeal defect and insufficient food intake [33], culture in axenic medium (ADR) [24] and dilution of bacteria on solid medium plate (sDR) [30]. All of these protocols extend C. elegans lifespan. It is of interest, and unexpected, that different forms of DR extend lifespan by different mechanisms $[3,32,34]$. For example, PHA-4, a FOXA transcription factor, is required for BDR-induced lifespan extension and for the longevity phenotype of eat-2 mutants [25]. In contrast, although DAF-16, a different FOXO transcription factor, is dispensable for BDR-mediated lifespan extension, it is essential for sDR treatment to increase C. elegans lifespan [30]. It was suggested that different dietary restriction protocols may require different effectors for lifespan extension, possibly due to the fact that some nutrients may be more 


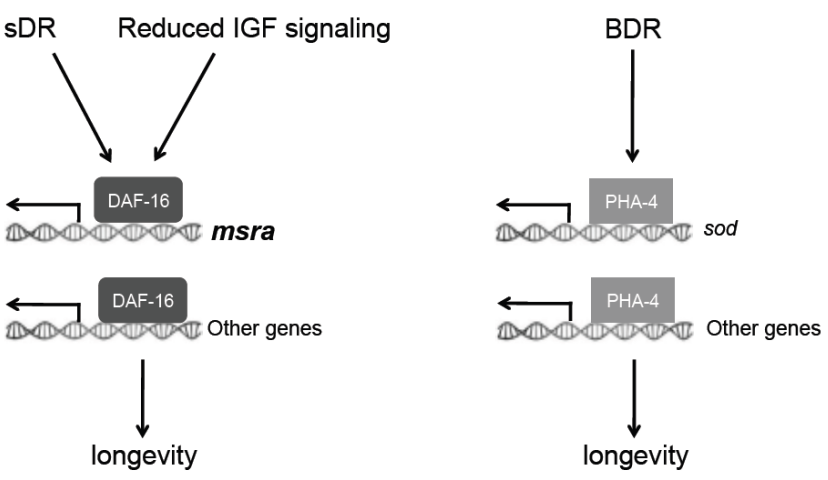

Figure 3: A model depicting a role of MSRA in dietary-restriction-induced longevity in $C$. elegans. As a target gene of DAF-16 FOXO transcription factor, MsrA is required for SDR-induced longevity, but is dispensable for BDR mediated lifespan extension. In addition to MsrA, other target genes of DAF16 are also required, especially for sDR- induced mean lifespan extension.

limiting than others depending on the DR method [34]. For example, sDR might reduce carbohydrates more severely than amino acids. Thus, $\mathrm{sDR}$ is dependent on FOXO that regulates carbohydrates metabolism. By contrast, another DR method BDR may mainly reduce amino acids. Therefore, BDR requires the FoxA/pha-4 transcription factor that has recently been found to be downstream of TOR (target-ofrapamycine) [35], the well-known amino-acid responsive pathway [36]. Interestingly, both PHA- 4 and DAF-16 regulate transcription of the antioxidant enzyme superoxide dismutase [25]. BDR involves the PHA4-dependent expression of sod-1, sod-2 and sod-5. DAF-16 regulates the expression of sod-1, sod-3 and sod-5 in response to reduced daf-2 insulin-like signaling [25].

Similar to SOD, expression of $m s r a-1$ is also positively regulated by DAF-16 [27]. When the daf-2 insulin-like signal is reduced or when C. elegans is under oxidative stress, the expression of msra-1 is upregulated dependent on DAF-16 [27]. The lifespan results presented here suggest that $m s r a-1$ is a major factor in the sDR-induced lifespan extension because it is a target gene of DAF-16 (Figure 3), since both DAF-16 and MsrA are required for sDR, but not BDR, to extend C. elegans lifespan. Interestingly, dietary restriction was found to alleviate abnormal locomotor activity and dopamine levels of MsrA-/- mice, suggesting that DR can prevent some of the oxidative damage seen in MsrA-/- mice [37], although in another study using MsrA-/- mice there was no evidence of abnormal locomotor activity [20]. In addition, the influence of MsrA on yeast lifespan was independent of DR (growth in the presence of $0.5 \%$ glucose) [23]. Recently, it was reported that yeast forkhead box transcription factors FKH1 and FKH2, putative DAF16 orthologs, are required for lifespan extension induced by severe caloric restriction (SCR) in which yeast cells are maintained in water [38]. However, the role of MsrA in these studies was not evaluated. The above results suggest different dietary restriction protocols may require different effectors for lifespan extension, as observed in C. elegans.

Since the mean lifespan of the sDR-treated msra-1 mutant increased slightly, it appears that other daf-16 target genes may also be required to achieve the full mean lifespan extension by sDR. It has been previously shown that msra-1 accounts for most of the longevity of daf- 2 mutants, and msra- 1 is certainly the major factor involved in the maximum lifespan extension in the present studies using sDR. Thus, msra-1, as a target gene of the DAF-16 FOXO transcription factor, is essential for life span extension conferred by both dietary restriction and reduction of the daf-2 insulin-like signaling pathway (Figure 3).

\section{Conclusions}

We provide genetic evidence that methionine sulfoxide reductase A (MsrA) plays an essential role in dietary restriction-mediated (sDR) lifespan extension in C. elegans. MsrA is an evolutionarily conserved antioxidant enzyme that has been found to be important for protecting cells against oxidative damage and regulating lifespan in several species. Interestingly, human Foxo3 positively regulates the expression of human MsrA and activates the C. elegans msra-1 promoter in human HEK293 cells [27]. This suggests $m s r a-1$ may be required for the healthbeneficial effect of dietary restriction in other species including humans.

\section{Summary}

Dietary restriction has been shown to increase the lifespan of many species varying from yeast to rodents. Methionine sulfoxide reductase $\mathrm{A}(\mathrm{MsrA})$ is a well-studied antioxidant enzyme that has been found to be important for protecting cells against oxidative damage and regulating lifespan in several species. However, the role of MsrA in dietary restriction has not been examined. Recently, an ortholog of MsrA (Msra-1) has been identified in Caenorhabditis elegans and it is required for the longevity phenotype of a mutant with reduced insulin-like signaling. Here we show that a loss-of-function mutation of msra-1 significantly suppresses the lifespan extension conferred by solid dietary restriction (sDR) in C. elegans. We also found that MsrA, like its positive regulator DAF-16/FOXO transcription factor, is dispensable for lifespan extension resulted from dietary restriction by diluted bacteria in liquid. These data suggest $m s r a-1$ is a major factor in the sDR-induced lifespan extension because it is a target gene of DAF16. This result, coupled with the previous finding that MsrA mediates the effect of insulin-like signaling on lifespan extension, indicates an essential role of MsrA in the aging process in C. elegans. Interestingly, human FOXO3a has been shown to regulate expression of $m s r a$ and can bind to the $C$. elegans msra-1 promoter, suggesting MsrA may be required for the health-beneficial effect of dietary restriction in other species including humans.

\section{Acknowledgements}

This work is supported by FONDECYT 1120213 to RA, FAU Research Priority Fund to H. W. and K.J., and by Ellison Medical Foundation New Aging Scholarship to K.J.

\section{References}

1. Anderson RM, Weindruch R (2012) The caloric restriction paradigm: implications for healthy human aging. Am J Hum Biol 24: 101-106.

2. Kenyon C (2005) The plasticity of aging: insights from long-lived mutants. Cell 120: 449-460.

3. Mair W, Dillin A (2008) Aging and survival: the genetics of life span extension by dietary restriction. Annu Rev Biochem 77: 727-754.

4. Sohal RS, Weindruch R (1996) Oxidative stress, caloric restriction, and aging Science 273: 59-63.

5. Sun J, Folk D, Bradley TJ, Tower J (2002) Induced overexpression of mitochondrial Mn-superoxide dismutase extends the life span of adult Drosophila melanogaster. Genetics 161: 661-672.

6. Schriner SE, Linford NJ, Martin GM, Treuting P, Ogburn CE, et al. (2005) Extension of murine life span by overexpression of catalase targeted to mitochondria. Science 308: 1909-1911.

7. Ruan H, Tang XD, Chen ML, Joiner ML, Sun G, et al. (2002) High-quality life extension by the enzyme peptide methionine sulfoxide reductase. Proc Natl Acad Sci U S A 99: 2748-2753.

8. Weissbach H, Resnick L, Brot N (2005) Methionine sulfoxide reductases: history and cellular role in protecting against oxidative damage. Biochim Biophys Acta 1703: 203-212. 
Citation: Minnerly J, Zhang J, Aldunate R, Weissbach H, Jia K (2013) Methionine Sulfoxide Reductase A Mediates Dietary Restriction-Induced Lifespan Extension in Caenorhabditis elegans. Aging Sci 1: 110. doi: 10.4172/2329-8847.1000110

9. Levine RL, Mosoni L, Berlett BS, Stadtman ER (1996) Methionine residues as endogenous antioxidants in proteins. Proc Natl Acad Sci U S A 93: 1503615040

10. Zhang $\mathrm{XH}$, Weissbach $\mathrm{H}$ (2008) Origin and evolution of the protein-repairing enzymes methionine sulphoxide reductases. Biol Rev Camb Philos Soc 83 249-257.

11. Moskovitz J, Poston JM, Berlett BS, Nosworthy NJ, Szczepanowski R, et al (2000) Identification and characterization of a putative active site for peptide methionine sulfoxide reductase (MsrA) and its substrate stereospecificity. J Bio Chem 275: 14167-14172

12. Sharov VS, Ferrington DA, Squier TC, Schöneich C (1999) Diastereoselective reduction of protein-bound methionine sulfoxide by methionine sulfoxide reductase. FEBS Lett 455: 247-250.

13. Grimaud R, Ezraty B, Mitchell JK, Lafitte D, Briand C, et al. (2001) Repair of oxidized proteins. Identification of a new methionine sulfoxide reductase. J Bio Chem 276: 48915-48920.

14. Lowther WT, Weissbach H, Etienne F, Brot N, Matthews BW (2002) The mirrored methionine sulfoxide reductases of Neisseria gonorrhoeae pilB. Nat Struct Biol 9: 348-352.

15. Kim HY, Gladyshev VN (2004) Methionine sulfoxide reduction in mammals: characterization of methionine-R-sulfoxide reductases. Mol Biol Cell 15: 10551064.

16. Moskovitz J, Rahman MA, Strassman J, Yancey SO, Kushner SR, et al. (1995) Escherichia coli peptide methionine sulfoxide reductase gene: regulation of expression and role in protecting against oxidative damage. J Bacteriol 177 502-507.

17. St John G, Brot N, Ruan J, Erdjument-Bromage H, Tempst P, et al. (2001) Peptide methionine sulfoxide reductase from Escherichia coli and Mycobacterium tuberculosis protects bacteria against oxidative damage from reactive nitrogen intermediates. Proc Natl Acad Sci U S A 98: 9901-9906.

18. Moskovitz J, Berlett BS, Poston JM, Stadtman ER (1997) The yeast peptidemethionine sulfoxide reductase functions as an antioxidant in vivo. Proc Nat Acad Sci U S A 94: 9585-9589.

19. Moskovitz J, Bar-Noy S, Williams WM, Requena J, Berlett BS, et al. (2001) Methionine sulfoxide reductase (MsrA) is a regulator of antioxidant defense and lifespan in mammals. Proc Natl Acad Sci U S A 98: 12920-12925.

20. Salmon AB, Pérez VI, Bokov A, Jernigan A, Kim G, et al. (2009) Lack of methionine sulfoxide reductase $A$ in mice increases sensitivity to oxidative stress but does not diminish life span. FASEB J 23: 3601-3608.

21. Picot CR, Perichon M, Cintrat JC, Friguet B, Petropoulos I (2004) The peptide methionine sulfoxide reductases, MsrA and MsrB (hCBS-1), are downregulated during replicative senescence of human WI-38 fibroblasts. FEBS Lett 558: 74 78 .

22. Yermolaieva O, Xu R, Schinstock C, Brot N, Weissbach H, et al. (2004) Methionine sulfoxide reductase A protects neuronal cells against brief hypoxia/ reoxygenation. Proc Natl Acad Sci U S A 101: 1159-1164.
23. Koc A, Gasch AP, Rutherford JC, Kim HY, Gladyshev VN (2004) Methionine sulfoxide reductase regulation of yeast lifespan reveals reactive oxygen species-dependent and -independent components of aging. Proc Natl Acad Sci U S A 101: 7999-8004

24. Houthoofd K, Braeckman BP, Johnson TE, Vanfleteren JR (2003) Life extension via dietary restriction is independent of the Ins/IGF-1 signalling pathway in Caenorhabditis elegans. Exp Gerontol 38: 947-954.

25. Panowski SH, Wolff S, Aguilaniu H, Durieux J, Dillin A (2007) PHA-4/Foxa mediates diet-restriction-induced longevity of $C$. elegans. Nature 447: 550-555.

26. Lee BC, Lee YK, Lee HJ, Stadtman ER, Lee KH, et al. (2005) Cloning and characterization of antioxidant enzyme methionine sulfoxide-S-reductase from Caenorhabditis elegans. Arch Biochem Biophys 434: 275-281.

27. Minniti AN, Cataldo R, Trigo C, Vasquez L, Mujica P, et al. (2009) Methionine sulfoxide reductase $A$ expression is regulated by the DAF-16/FOXO pathway in Caenorhabditis elegans. Aging Cell 8: 690-705.

28. Brenner S (1974) The genetics of Caenorhabditis elegans. Genetics 77: 71-94

29. Williams BD, Schrank B, Huynh C, Shownkeen R, Waterston RH (1992) A genetic mapping system in Caenorhabditis elegans based on polymorphic sequence-tagged sites. Genetics 131: 609-624.

30. Greer EL, Dowlatshahi D, Banko MR, Villen J, Hoang K, et al. (2007) An AMPKFOXO pathway mediates longevity induced by a novel method of dietary restriction in C. elegans. Curr Biol 17: 1646-1656.

31. Klass MR (1977) Aging in the nematode Caenorhabditis elegans: major biological and environmental factors influencing life span. Mech Ageing Dev 6: 413-429.

32. Mair W, Panowski SH, Shaw RJ, Dillin A (2009) Optimizing dietary restriction for genetic epistasis analysis and gene discovery in C. elegans. PLoS One 4 e4535.

33. Lakowski B, Hekimi S (1998) The genetics of caloric restriction in Caenorhabditis elegans. Proc Natl Acad Sci U S A 95: 13091-13096.

34. Greer EL, Brunet A (2009) Different dietary restriction regimens extend lifespan by both independent and overlapping genetic pathways in $C$. elegans. Aging Cell 8: 113-127.

35. Sheaffer KL, Updike DL, Mango SE (2008) The Target of Rapamycin pathway antagonizes pha-4/FoxA to control development and aging. Curr Biol 18: 1355 1364.

36. Avruch J, Hara K, Lin Y, Liu M, Long X, et al. (2006) Insulin and amino-acid regulation of mTOR signaling and kinase activity through the Rheb GTPase. Oncogene 25: 6361-6372.

37. Oien DB, Osterhaus GL, Lundquist BL, Fowler SC, Moskovitz J (2010) Caloric restriction alleviates abnormal locomotor activity and dopamine levels in the brain of the methionine sulfoxide reductase A knockout mouse. Neurosci Let 468: 38-41.

38. Postnikoff SD, Malo ME, Wong B, Harkness TA (2012) The yeast forkhead transcription factors fkh1 and fkh2 regulate lifespan and stress response together with the anaphase-promoting complex. PLoS Genet 8: e1002583. 